\title{
BENFOTHIAMINE ADMINISTRATION IN PATIENTS WITH DILATED CARDIOMYOPATHY AND ADVANCED HEART FAILURE WITH CHRONIC DIURETIC TREATMENT
}

\author{
DANA-ADRIANA ILUȚIU-VARVARA ${ }^{\mathrm{a}}$, DAN RĂDULESCU ${ }^{\mathrm{b} *}$, \\ VALER DONCA ${ }^{\mathrm{b}}$
}

\begin{abstract}
It is well known that long term administration of high dose loop diuretics in patients with heart failure, may in some cases aggravate the cardiac failure symptoms. Part of this effect may be due to thiamine depletion secondary to diuretics use. The aim of our present study was to assess whether administration of benfothiamine in patients with advanced congestive cardiac failure treated on long term with high dose loop diuretics, can prevent the alteration of heart failure.
\end{abstract}

Keywords: benfothiamine, cardiovascular diseases, cardiomyopathy, diuretic treatment, heart failure, thiamine.

\section{INTRODUCTION}

Thiamine (Fig. 1) is a water soluble vitamin. The best-characterized form is thiamine pyrophosphate (TPP), a coenzyme in the catabolism of sugars and amino acids. Thiamine is present in meat and cereals. Thiamine pyrophosphate is a key enzyme in carbohydrates metabolism. It results from thiamine and ATP in kidneys, liver, blood cells. In thiamine depletion states, the hexose monophosphate and citric acid cycles are affected. These states are secondary either to poor nutrition (rice), or to chronic alcohol intake, hyperthyroidism, diarrhea, diuretics. Thiamine is present in tissues, at least in 3 forms (mono-, di and triphosphate). In humans, thiamines exist especially

\footnotetext{
a Universitatea Tehnică din Cluj-Napoca, Facultatea de Instalaţii, Str. Memorandumului, Nr. 28, RO-400114 Cluj-Napoca, Romania

b Universitatea de Medicină şi Farmacie Iuliu Hațieganu, Facultatea de Medicină, Str. Emil Isac, Nr. 13, RO-400023 Cluj-Napoca, Romania

*Corresponding author:dan_rad31@yahoo.com
} 
as thiamine diphosphate, more than two thirds being present in erhytrocytes. A low erythrocyte transketolase activity is a marker of thiamine deficiency. In general, we appreciate the serum diphosphate activity, by measuring the thiamine pyrophosphate effect (over $15 \%$ in thiamine deficiency). Some researchers measure the erythrocyte thiamine diphosphate, while others measure thiamine levels by using high performance liquid chromatography $[1,2,3]$.

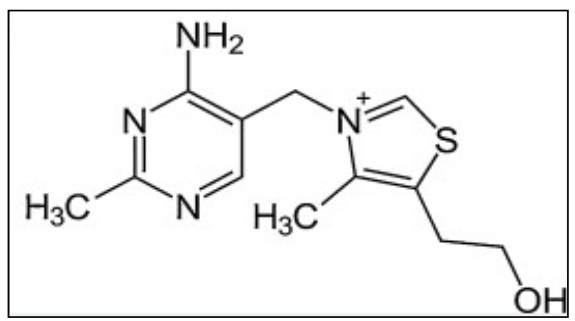

Figure 1. Chemical structure of thiamine

Thiamine (Fig. 2), as well as its phosphate derivatives, can also be detected directly in whole blood, tissues, foods, animal feed, and pharmaceutical preparations following the conversion of thiamine to fluorescent thiochrome derivatives (Thiochrome Assay) and separation by high-performance liquid chromatography (HPLC) [4].

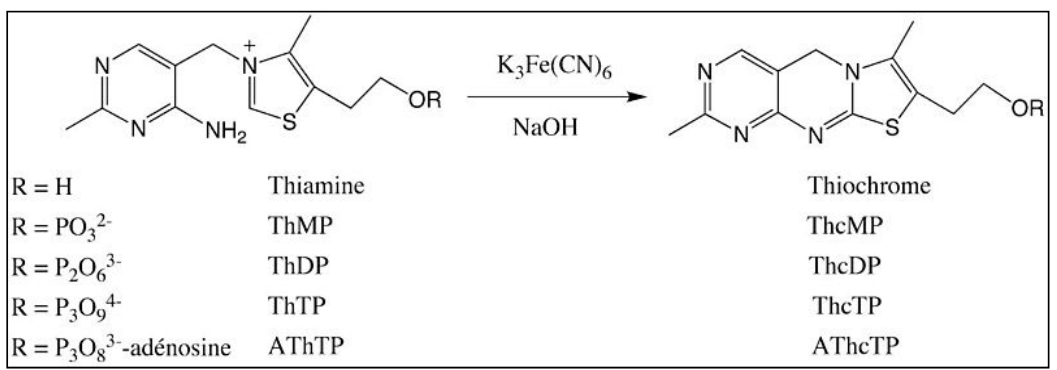

Figure 2. Detection of Thiamine through Thiochrome Assay $[4,5]$

Severe thiamine depletion generates cardiac failure (wet beriberi), but recent studies documented left ventricular insufficiency even after moderate thiamine depletion, in patients receiving loop diuretics [5]. 
In a study on 23 cardiac failure patients treated with furosemide over a period of 3 to 14 months, was observed a serum thiamine depletion (by measuring thiamine pyrophosphate effect), secondary to high urinary thiamine excretion. After oral thiamine supplementation in these patients, was reported an increase in left ventricular ejection fraction, in NYHA exercise capacity, in systolic and diastolic pressures, in these patients. In another study, in 30 patients with heart failure treated longer periods with furosemide, Shimon et al. documented a $22 \%$ rise in left ventricular ejection fraction after thiamine administration. Plasma thiamine which was low after diuretic therapy, increased after thiamine administered intravenous. After intravenous thiamine, thiamine pyrophosphate levels decreased in the thiamine supplementation arm in comparison to the placebo one [6].

For thiamine supplementation to be efficient, high levels of this water soluble vitamin in tissues are required. Fujiwara et al., [7] discovered in vegetables a class of lipid soluble thiamines (allithiamines), of which benfothiamine has the highest bioavailability. After oral administration benfothiamine generates considerably higher thiamine concentrations (at least five times greater) in tissues, than the water soluble thiamine. Benfothiamine has an open thiazole ring, which closes once benfothiamine is absorbed passively through intestinal mucosa, generating active thiamine. Benfothiamine is available in a proportion of at least $25 \%$, for 8 days in tissues, much longer than water soluble thiamine [8,9]. It is very efficient in the treatment of macrovascular and microvascular endothelial dysfunction and oxidative stress, in patients with diabetes mellitus and secondary complications [10,11, 12].

The first lipophilic thiamine derivative was isolated from garlic (Allium sativum) extracts in the early 1950s [7]. It is an allyl disulfide derivative called allithiamine (Fig. 3). Since then, several analogs of this molecule were synthesized with the hope that they would be better absorbed and have a higher bioavailability than thiamine hydrochloride or mononitrate $[13,14]$.

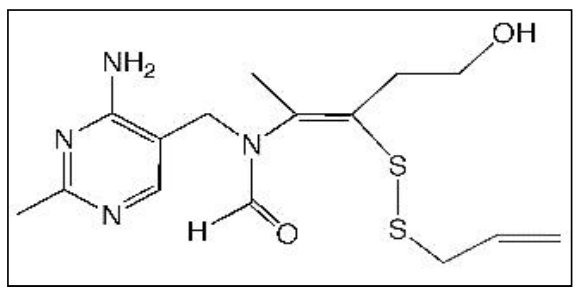

Figure 3. Chemical structure of allithiamine (thiamine allyl disulfide)

Benfotiamine (Fig. 4) is a third derivative with better bioavailability than thiamine. In contrast to the above-mentioned derivatives it is not a disulfide but an S-acyl derivative. 


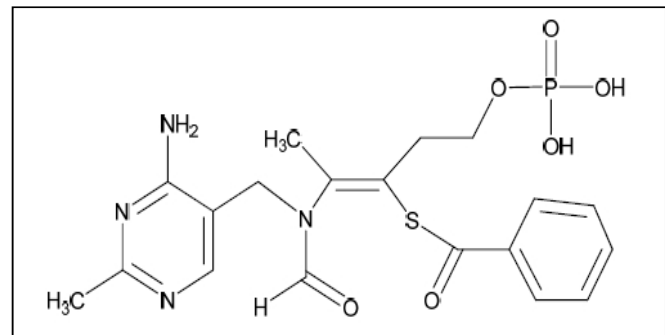

Figure 4. Chemical structure of benfothiamine

( $\mathrm{S}$ - benzoylthiamine $\mathrm{O}$ - monophosphate)

Benfothiamine can also be detected directly in whole blood, tissues, foods, animal feed, and pharmaceutical preparations after derivatization, through chemical oxidation with ferricyanide at $\mathrm{pH} 13$ to form fluorescent thiochrome. Fluorimetric detection is carried out in conjunction with dispersive liquid - liquid microextraction (DLLME) coupled to liquid chromatography (LC) [15].

The purpose of our paper was to assess whether administration of benfothiamine in patients with advanced congestive cardiac failure treated on long term with high dose loop diuretics, can prevent the aggravation of heart failure.

\section{RESULTS AND DISCUSSION}

In Table 1 are presented the parameters of both groups at the beginning of the study. As can be seen the parameters are very close between groups.

Table 1. Patients data at the start of treatment

\begin{tabular}{|c|c|c|}
\hline Data & Study group (thiamine) & Control group \\
\hline Number of patients & 20 & 20 \\
\hline Age (year) & $64 \pm$ & $66 \pm$ \\
\hline NYHA class & $3.1 \pm 0.7$ & $3.3 \pm 0.8$ \\
\hline Diuretic (furosemid mg/day) & $100 \pm 20$ & $100 \pm 40$ \\
\hline Systolic BP (mmHg) & $100 \pm 14$ & $104 \pm 16$ \\
\hline EF (\%) & $24 \pm 8$ & $26 \pm 10$ \\
\hline Cardiac output (l/min) & $3.2 \pm 0.6$ & $3.4 \pm 0.8$ \\
\hline Diuresis (I/day) & $1.44 \pm 0.4$ & $1.64 \pm 0.6$ \\
\hline NTproBNP (pg/ml) & $3860 \pm 838$ & $4020 \pm 926$ \\
\hline
\end{tabular}


study.

In Table 2 are the same parameters at three months, at the end of

Table 2. Patients data after three months of treatment

\begin{tabular}{|c|c|c|}
\hline Data & Study group (thiamine) & Control group \\
\hline Number of patients & 10 & 10 \\
\hline Age (year) & $64 \pm$ & $66 \pm$ \\
\hline NYHA class & $2.6 \pm 0.6$ & $3.4 \pm 0.6$ \\
\hline Diuretic (furosemid mg/day) & $80 \pm 30$ & $80 \pm 40$ \\
\hline Systolic BP $(\mathrm{mmHg})$ & $99 \pm 12$ & $102 \pm 14$ \\
\hline EF $(\%)$ & $28 \pm 9$ & $25 \pm 8$ \\
\hline Cardiac output (l/min) & $3.3 \pm 0.7$ & $3.5 \pm 0.9$ \\
\hline Diuresis (l/day) & $2.20 \pm 0.7$ & $1.70 \pm 0.5$ \\
\hline NTproBNP $(\mathrm{pg} / \mathrm{ml})$ & $1020 \pm 222$ & $2800 \pm 428$ \\
\hline
\end{tabular}

As can be seen, we documented, in the study group, after three months of thiamine supplementation, amelioration of the NYHA class with a higher left ventricular ejection fraction, a higher diuresis. At three months we found significantly lower levels of NT proBNP (brain natriuretic peptide) in the study group, compared to the control group. There were no significant differences concerning systolic blood pressure and cardiac output variation, between the two groups at the end of study.

After 3 months of benfothiamine administration, we documented in the study group an amelioration of the NYHA class with a higher left ventricular ejection fraction, a higher diuresis and especially lower levels of NT proBNP, compared with the control group. There were no significant differences concerning systolic blood pressure and cardiac output variation, between the two groups. We noticed no adverse effects of benfothiamine administration (hypotension, gastrointestinal disturbances, as some others reported).

Different studies on streptozocin-induced type 2 diabetes in murine models have shown that benfotiamine prevented ventricular diastolic dysfunction and heart failure through Akt/Pim-1 mediated survival pathway. By preventing cardiomyocyte apoptosis, myocardial fibrosis and microvascular rarefaction in diabetic experimental hearts, benfotiamine improved their ventricular performance [16]. Other studies documented an improvement of left ventricular systolic performance in clinical and experimental models of heart failure after benfotiamine administration, whereas the drug had no effect in healthy hearts $[16,17]$. Benfotiamine, a transketolase activator, directing glucose to the pentose phosphate pathway, improves experimentally, the functional recovery of infarcted murine hearts, by activation of pro-survival G6PD/Akt signalling pathway and modulation of neurohormonal response [18]. 
The liposoluble benfotiamine is superior to thiamine as it inhibits formation of advanced glycation end products not only in diabetic but also in aging organisms. As it is well known, glycation produces renal, nerve and retinal damage and also cardiovascular disease. As a consequence, benfotiamine may prevent or even treat neuropathies, blindness, renal failure, cardiomyopathy, arterial peripheral vascular disease. Treatment with thiamine did not significantly affect the formation of advanced glycation end products and as a consequence thiamine cannot prevent the mentioned diseases $[19,20]$. Deficiency of thiamine, like in beriberi, produces an increased angiogenesis. Benfothiamine increases significantly the angiogenesis. As opposed to thiamine which cannot help in Alzheimer disease, benfotiamine improves the condition of Alzheimer patients and also reduces amyloid formation [18].

As it is well known, thiamine deficiency is associated with oxidative stress. Oxidative stress affects the blood-brain barrier, mitochondrial integrity, nucleic acid and neural stem cell function, induces neuroinflamation and apoptosis. Secondary to producing reactive oxygen species and diminishing the generation of antioxidants, hyperglycemia causes oxidative stress. Benfotiamine diminishes superoxide and hydroxyl radical levels in diabetic and aging hearts by regenerating the antioxidant NADPH, secondary to the activation of the pentose phosphate shunt. By preventing advanced glycation end products accumulation, benfotiamine prevents myocardial interstitial fibrosis $[21,22,23]$.

There were some limits of our study: the number of patients was not very large; as the patients had advanced heart failure, we could not practice exercise test, in order to better quantify the left ventricular failure, than by simple clinical NYHA class.

\section{CONCLUSIONS}

After 3 months of benfothiamine supplementation, we documented an improved clinical status, left ventricular ejection fraction, cardiac output in patients with advanced heart failure treated with high dose furosemid. Also serum NT proBNP decreased considerably in this group of patients.

\section{EXPERIMENTAL SECTION}

The study group comprised 20 patients with dilated cardiomyopathy (DCM) and advanced heart failure, on standard therapy and high dose furosemid under oral thiamine supplementation $200 \mathrm{mg} /$ day, monitored for three months. The control group comprised gender and aged matched similar patients with DCM and severe heart failure, treated with standard therapy 
and high dose furosemid, but without oral thiamine. Both groups were treated with furosemid $80 \mathrm{mg}$ and over daily, orally, also in the last three months and continued it during at least three months, till the end of study. All 40 patients were suffering from primitive or ischemic DCM. Patients with recent myocardial infarction, atrial fibrillation, valvulopathies, patients with left ventricular ejection fraction over $40 \%$, were excluded. We also excluded patients on other diuretics than furosemid and patients thiamin deficient of other causes (neoplasia, alcoholism, malnutrition, infections, thyroid, liver or renal diseases, diabetus mellitus). study:

Cardiac function was assessed clinically during the three months

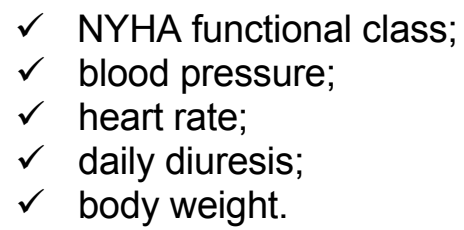

For ultrasound examination an Aloka echocardiograph with a 3.5 $\mathrm{MHz}$ transducer and Doppler mode was used, the examination being performed in conformity with the guidelines of the American Society of Echocardiography. The left ventricular systolic performance was assessed by measuring the global EF, monthly, based on apical 4 chambers view according to ASE. Cardiac output was measured using Doppler at left ventricular outflow tract.

Serum brain natriuretic peptide NT proBNP was determined by immunoassay in each patient at the beginning and at the end of the study (normal value $20-40 \mathrm{pg} / \mathrm{ml}$ ).

We used an unpaired test for normally distributed variables (respectively the Wilcoxon two sample tests for skewed distributions) for comparison between 2 groups. We reported normally distributed variables as mean \pm SD, and the skewed distributed as median and range. We used Pearson's coefficient test to assess correlations between variables and considered $p<0.05$ significant.

\section{REFERENCES}

1. D. Loew, International Journal of Clinical Pharmacology and Therapeutics, 1996, 34, 47.

2. Howard Smithline, M. Donnino, D.J. Greenblatt, BMC Clinical Pharmacology, 2012, 12, 4.

3. K.T. Weber, Journal of the American College of Cardiology, 2004, 44, 1308. 
4. J. Lu, E.L. Frank, Clinical Chemistry, 2008, 54, 901.

5. D. Leslie, M. Gheorghiade, American Heart Journal, 1996, 131, 1.

6. Shimon, S. Almog, Z. Vered, H. Seligmann, M. Shefi, E. Peleg, American Journal of Medicine, 1995, 98, 485.

7. M. Fujiwara, H. Watanabe, K. Matsui, Journal of Biochemistry, 1954, 41, 29.

8. P. Balakumar, A. Rohilla, P. Krishan, P. Solairaj, A. Thangathirupathi, Pharmacological Research, 2010, 61, 482.

9. M.L. Volvert, S. Seyen, M. Piette, B. Evrard, M. Gangolf, J.C. Plumier, L. Bettendorff, BMC Clinical Pharmacology, 2008, 8, 10.

10. Stirban, M. Negrean, B. Stratmann, Diabetes Care, 2006, 29, 2064.

11. Stirban, M. Negrean, B. Stratmann, Diabetes Care, 2007, 30, 10.

12. D.A. Fraser, N.P. Hessvik, N. Nikolić, V. Aas, K.F. Hanssen, S.K. Bøhn, G.H. Thoresen, A.C. Rustan, Genes \& Nutrition, 2012, 7, 459.

13. Greb, R. Bitsch, International Journal of Clinical Pharmacology and Therapeutics, 1998, 36, 216.

14. D. Lonsdale, Evidence Based Complementary and Alternative Medicine, 2006, $3,49$.

15. P. Vinas, M. Bravo-Bravo, I. Lopez-Garcia, M. Hernandez-Cordoba, Analytical Methods, 2012, 4, 2759.

16. R.G. Katare, A. Caporali, A. Oikawa, M. Meloni, C. Emanurli, P. Madeddu, Circulation Heart Failure, 2010, 3, 294.

17. J. DiNicolantonio, C.J. Lavie, A.K. Niazi, J.H. O’Keefe, T. Hu, Ochsner Journal, 2013, 13, 495.

18. R. Katare, A. Caporali, C Emanueli, P. Madeddu, Journal of Molecular and Cellular Cardiology, 2010, 49, 625.

19. H. Stracke, H.P. Hammes, D. Werkmann, K. Mavrakis, I. Bitsch, M. Netzel, Experimental and Clinical Endocrinology \& Diabetes, 2001, 109, 330.

20. M.L. Volvert, S. Seven, M. Piette, B. Evrard, M. Gangolf, J.C. Plumier, BMC Pharmacology, 2008, 12, 8.

21. S. Hazell, S. Faim, G. Wertheimer, V. R. Silva, C. S. Marques, Neurochemistry International, 2013, 62, 796.

22. Stirban, M. Negrean, B. Stratmann, T. Gawlowski, T. Horstmann, C. Gotting, Diabetes Care, 2006, 29, 2064.

23. Nenna, F. Nappi, S.S. Avtaar Singh, F.W. Sutherland, F. Di Domenico, M. Chello, C. Spadaccio, Research in Cardiovascular Medicine, 2015, 23, 2. 